\title{
FRAGEN AUS DER PRAXIS
}

Werte Kolleginnen und Kollegen, an dieser Stelle beantworten wir Ihre Fragen, die für alle Leser interessant sein könnten. Scheuen Sie also nicht davor zurück, uns Ihre Fragen zu senden. Für Mitglieder steht unsere Mitgliederbetreuung für Humanmediziner und für Zahnärzte gerne zurVerfügung (siehe unterVereinsinformation)!

Ihre Redaktion

\section{Frau Dr. Barbara H. aus München fragt:}

Ich habe gerade einen der kommenden Ohrkurse der Stufe 2 gebucht. Und ich habe gehört, dass man dort den diagnostischen Puls lernen kann, der in den Büchern RAC heißt oder auch Nogier-Puls. Ich war schon neugierig und habe im Großen Buch der Ohrakupunktur nachgelesen, was das ist. Es klingt spannend, dass man dann über die sogenannten Störherdhinweispunkte am Ohr eine schnelle Einschätzung der Anzahl und Stärke der Störherde bekommen soll. Und dass man noch schneller finden kann, wo der stärkste Störherd sich versteckt. Auch habe ich gelesen, dass man sogar Störherde finden kann, die ich im Anamnesebogen als Möglichkeit gar nicht hätte erkennen können, also z.B. Narben, die mir der Patient nicht angibt oder vielleicht vergessen hat?

Bis jetzt bin ich begeistert in meiner Praxis mit dem Punktsuchgerät zugange - und meine Erfolge können sich sehen lassen (Abb. 1). Meine Frage: Werde ich nach dem Ohrkurs der Stufe 2 das Punktsuchgerät vielleicht nicht mehr brauchen? Ich habe aber auch schon gehört, dass auf diesen Kursen empfohlen wird, das Gerät in jedem Fall zu behalten? Für eine kurze Information hierzu wäre ich sehr dankbar.

\section{Antwort:}

Es ist richtig, wir empfehlen, das Punktsuchgerät zu behalten. Gerade am Anfang, wenn man das RAC-Tasten noch üben muss oder möchte, ist das Punktsuchgerät ein verlässlicher Begleiter und Trainer, der den gefundenen Punkt objektivieren kann. Andersherum kann man auch an einem mittels Punktsuchgerät gefundenen Ohrpunkt den RAC üben, wissend, dass dieser Punkt existiert und an dieser Stelle ein Nogier-Pulsreflex ausgelöst werden muss.

Bitte nicht vergessen, dass Sie unbedingt nach dem Besuch von Ohr 2 einen Hospitationskurs besuchen (akupunktur-seminare.de), um unter Anleitung praktisch zu arbeiten. Selbst für Kollegen, die den RAC schon sehr gut beherrschen: Auch wenn man einmal müde, gestresst oder nicht so konzentriert ist, und deshalb den RAC nicht so gut tasten kann, ist das Punktsuchgerät eine verlässliche objektive Hilfe, die Sie nicht im Stich lässt. Auch wenn Sie einmal wegen Schlafmangel oder zu viel Alkohol am Vorabend den RAC vielleicht nicht so zuverlässig tasten können, können Sie mit Ihrem Punktsuchgerät in jedem Fall eine Diagnostik über die Ohrreflexzonen durch führen.

Deshalb: Auch wenn Sie den RAC schnell erlernt haben oder bereits gut beherrschen, geben Sie Ihr Punktsuchgerät nicht weg!

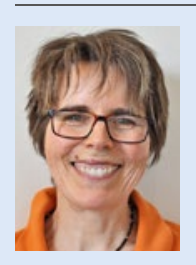

Den Fragekasten bearbeitet hat

Dr. med. Beate Strittmatter

FA für Allgemeinmedizin, Akupunktur, Sportmedizin, Naturheilverfahren, Ausbildungsleitung Ohrakupunktur

Quellenstr.19, 66121 Saarbrücken

Strittmatter@t-online.de 